\title{
Registro de Thaumastocoris peregrinus Carpintero e Dellapé na Região Sudoeste do Paraná
}

\author{
Grasielle Adriane Toscan Lorencetti ${ }^{1}$, Michele Potrich ${ }^{2}$, \\ Everton Ricardi Lozano da Silva², Sérgio Miguel Mazaro³, \\ Leonardo Rodrigues Barbosa ${ }^{4}$
}

\begin{abstract}
${ }^{1}$ Programa de Pós-Graduação em Agronomia - PPGAG, Universidade Tecnológica Federal do Paraná - UTFPR, Pato Branco/PR, Brasil

${ }^{2}$ Coordenação de Ciências Biológicas, Universidade Tecnológica Federal do Paraná - UTFPR, Dois Vizinhos/PR, Brasil ${ }^{3}$ Coordenação de Agronomia - COAGR, Universidade Tecnológica Federal do Paraná - UTFPR, Dois Vizinhos/PR, Brasil ${ }^{4}$ Laboratório de Entomologia Florestal, Embrapa Florestas, Colombo/PR, Brasil
\end{abstract}

\section{RESUMO}

Em junho de 2012 foi registrada a ocorrência do percevejo bronzeado do eucalipto, Thaumastocoris peregrinus, na região sudoeste do Paraná, atacando árvores de Eucalyptus camaldulensis Dehn. O registro foi realizado no município de Realeza (coordenadas UTM SAD 69: 245000 E 7153682S).

Palavras-chave: percevejo bronzeado do eucalipto, monitoramento, ocorrência.

\section{Record of Thaumastocoris peregrinus in the Southwest Region of Paraná State, Brazil}

\begin{abstract}
In June 2012, the occurrence of bronze bug of eucalyptus, Thaumastocoris peregrinus, was recorded in the southwest region of Paraná state, attacking trees of Eucalyptus camaldulensis Dehn (Myrtaceae). The occurrence was recorded in the municipality of Realeza (SAD 69 Coordinates UTM: 245000 E; 7153682 S).
\end{abstract}

Keywords: eucalyptus bronze bug, monitoring, occurrence.

O gênero Thaumastocoris, descrito por Kirkaldy em 1908, possui cinco espécies conhecidas, sendo que Thaumastocoris peregrinus Carpintero \& Dellapé (2006) (Hemiptera, Thaumastocoridae) é a única espécie detectada fora da Austrália (Carpintero \& Dellapé, 2006). O inseto adulto apresenta cerca de $3 \mathrm{~mm}$ de comprimento, corpo achatado e cor marrom-clara. Os ovos são pretos, achatados, com a região central côncava. As ninfas são claras e vão escurecendo com o tempo. Cada fêmea coloca em média 60 ovos (Jacobs \& Nesser, 2005; Carpintero \& Dellapé, 2006; Wilcken et al., 2010).
A duração do período embrionário dos ovos é de cerca de seis dias. A fase ninfal compreende cinco instares, com duração de até 16 dias. A longevidade dos adultos varia de 14 a 42 dias (Soliman et al., 2012).

Esse inseto se alimenta da seiva das folhas de algumas espécies dos gêneros Eucalyptus e Corymbia. As folhas inicialmente sofrem um prateamento, passando para tons de marrom e vermelho, o que confere à árvore um aspecto bronzeado (Jacobs \& Nesser, 2005; Wilcken et al., 2010). Esse processo diminui a taxa fotossintética da planta, culminando com o secamento 
e a queda das folhas, com consequente prejuízo ao seu desenvolvimento (Jacobs \& Nesser, 2005). Além disso, esse processo fragiliza a planta, propiciando a entrada de outros agentes fitopatogênicos.

T. peregrinus está descrito em vários locais no mundo (Jacobs \& Nesser, 2005; Carpintero \& Dellapé, 2006; Martínez \& Bianchi, 2010; Laudonia \& Sasso, 2012; Garcia et al., 2013). No Brasil, a ocorrência desse inseto foi relatada pela primeira vez em maio de 2008, por Wilcken et al. (2010), no município de São Francisco de Assis, Rio Grande do Sul, atacando clones híbridos de Eucalyptus grandis Hill ex Maiden x Eucalyptus urophylla S. T. Blake. Em menos de um ano foi encontrado em mais nove municípios desse estado (Wilcken et al., 2010). Em dezembro de 2008, foi confirmado em Minas Gerais (Wilcken et al., 2010). Em abril de 2009 foi identificado no estado de Santa Catarina (Savaris et al., 2011), em julho de 2009 no estado do Espírito Santo, em outubro de 2009 no estado do Rio de Janeiro e no estado de Mato Grosso do Sul (Wilcken et al., 2010). Nesse mesmo ano, o inseto já estava disseminado em 74 municípios do estado de São Paulo e foi registrado pela primeira vez no estado do Paraná, próximo à cidade de Curitiba (Wilcken et al., 2010; Barbosa et al., 2010). Em 2011 foi detectado no estado de Goiás (Pereira et al., 2013).

No estado do Paraná, a região sudoeste apresenta potencial para o aumento no plantio de eucalipto. A demanda por madeira para energia, impulsionada principalmente pelo setor avícola, vem alavancando a produção. Até o momento, essa região, que engloba 42 municípios e tem uma extensão de $17.102 .750 \mathrm{~km}^{2}$ (AMSOP, 2012), não havia constatado a ocorrência de T. peregrinus. Em junho de 2012, no entanto, foi detectado infestação do percevejo no município de Realeza, em uma propriedade rural às margens da Rodovia PR - 182 (coordenadas UTM SAD 69: 245000 E 7153682 S, altitude: 396 metros). O foco de infestação foram árvores de E. camaldulensis Dehn., plantadas há, aproximadamente, cinco anos, sendo que algumas apresentavam sinais de danos severos. Também se observaram inseto e ovos por toda a planta de eucalipto, nos vários estratos, e segundo Jacobs \& Nesser (2005), a maior ênfase de infestação é na copa das árvores.

No mesmo local ainda foram encontrados insetos, em menor número, atacando Eucalyptus dunnii Maiden e E. grandis. De acordo com Noack \& Coviella (2006), quando fortemente infestadas, as folhas exibem um prateamento e avermelhamento e conforme a infestação progride a copa inteira fica amarelo-avermelhada e a árvore perde as folhas.

Exemplares do inseto, exemplares de ovos e ramos atacados foram coletados do terço inferior das árvores, armazenados em sacos plásticos e levados para o Laboratório de Controle Biológico da Universidade Tecnológica Federal do Paraná, Campus Dois Vizinhos, para identificação dos insetos. Exemplares foram fixados em álcool 70\%, para observação detalhada com auxílio de microscópio estereoscópio. A identificação foi realizada de acordo com Carpintero \& Dellapé (2006) e Wilcken et al. (2010).

Observou-se que após as frentes frias que ocorreram em junho de 2012 houve drástica redução na infestação, no entanto deve-se considerar ações para priorizar o monitoramento, manejo e orientação ao plantio de espécies e clones de Eucalyptus e Corymbia resistentes, a fim de evitar a disseminação do inseto.

A espécie E. camaldulensis é considerada de grande importância e alta resistência ao déficit hídrico, no entanto Wilcken (2008) classificou E. camaldulensis como uma espécie com alta incidência de pragas, o que pode comprometer a atividade florestal no Brasil. E. camaldulensis, Eucalyptus viminalis Labill., E. grandis, Eucalyptus terecticornis Smith., Eucalyptus smithii R. T. Baker, E. grandis X E. camaldulensis e E. grandis $\mathrm{X}$ Eucalyptus urophylla foram indicadas como as espécies mais susceptíveis aos danos causados por T. peregrinus (Wilcken et al., 2009). Menezes et al. (2011), testando a preferência alimentar de T. peregrinus sobre 14 espécies de Eucalyptus e uma espécie de Corymbia, em laboratório, confirmaram a preferência desse inseto pela espécie E. camaldulensis, porém não descartando a possibilidade de ataque a Corymbia citriodora (Hill \& Johnson).

$\mathrm{O}$ controle químico é realizado em pequena escala na Austrália, porém torna-se inviável face à dimensão e aos processos de certificação florestal que norteiam plantios comerciais. Pesquisas contemplam o uso do controle biológico, com predadores, parasitoides e fungos entomopatogênicos no Brasil, sendo que a importação do parasitoide de ovos Cleruchoides noackae Lin. e Huber (Hymenoptera: Mymaridae) e suas liberações têm demonstrado potencial de controle em diversas áreas em que T. peregrinus vem ocorrendo (Barbosa, informação pessoal ${ }^{1}$ ).

\footnotetext{
${ }^{1}$ Dr. Leonardo Rodrigues Barbosa. Pesquisador A da Empresa Brasileira de Pesquisa Agropecuária - Embrapa. CNPF - Centro Nacional de Pesquisa de Florestas.
} 
Nesse sentido, trabalhos que informem a ocorrência de T. peregrinus e seu alto potencial de dispersão auxiliam na prospecção do monitoramento e desenvolvimento de programas de controle. O registro de novas regiões com ocorrência do inseto evidencia a necessidade de melhores métodos de proteção e manejo dessa praga, como a implementação de medidas fitossanitárias no transporte de mudas e madeira de Eucalyptus, além de campanhas informativas quanto ao uso de espécies menos suscetíveis. A expansão da área de atuação serve de alerta para a ameaça que T. peregrinus representa para os plantios de Eucalyptus no Brasil.

\section{STATUS DA SUBMISSÃO}

Recebido: 7 out., 2014

Aceito: 21 mar., 2015

\section{AUTOR(ES) PARA CORRESPONDÊNCIA}

\section{Michele Potrich}

Coordenação de Ciências Biológicas, Universidade Tecnológica Federal do Paraná UTFPR, Estrada para Boa Esperança, Km 04, Comunidade São Cristóvão, CEP 85660-000, Dois Vizinhos, PR, Brasil

e-mail: michelepotrich@utfpr.edu.br

\section{REFERÊNCIAS}

Associação dos Municípios do Sudoeste do Paraná AMSOP. [cited 2012 June 16]. Available from: http:// www.amsop.com.br/home.php.

Barbosa LR, Santos F, Wilcken CF, Soliman EP. Registro de Thaumastocoris peregrinus (Hemiptera, Thaumastocoridae) no Estado do Paraná. Nota Científica. Pesquisa Florestal Brasileira 2010; 30(61): 75-77. http://dx.doi.org/10.4336/2010. pfb.30.61.75.

Carpintero DL, Dellapé PM. A new species of Thaumastocoris Kirkaldy from Argentina (Heteroptera: Thaumastocoridae: Thaumastocorinae). Zootaxa 2006; 1228: 61-68.

Garcia A, Figueiredo E, Valente C, Monserrat VJ, Branco M. First record of Thaumastocoris peregrinus in Portugal and of the neotropical predator Hemerobius bolivari in Europe. Bulletin of Insectology 2013; 66(2): 251-256.

Jacobs DH, Nesser S. Thaumastocoris australicus Kirkaldy (Hetereptera: Thaumastocoridae): a new insect arrival in Sounth Africa, damaging to Eucalyptus trees. South African Journal of Science 2005; 101(5-6): 233-236.
Laudonia S, Sasso R. The bronze bug Thaumastocoris peregrinus: a new insect recorded in Italy, damaging to Eucalyptus trees. Bulletin of Insectology 2012; 65(1): 89-93.

Martínez G, Bianchi M. Primer registro para Uruguay de lachinche del eucalipto, Thaumastocoris peregrinus Carpintero\&Dellappé, 2006 (Heteroptera: Thaumastocoridae). Agrociencia 2010; 14(1): 15-18.

Menezes MJS, Lorencetti GAT, Dallacorte S, Oliveira TM, Potrich M, Silva ERL. Preferência alimentar de Thaumastocoris peregrinus Carpintero\&Dellapé (Hemiptera: Thaumastocoridae) a diferentes espécies do gênero Eucalyptus. In: I Congresso de Ciência e Tecnologia da UTFPR; 2011; Dois Vizinhos. Dois Vizinhos: UTFPR; 2011. p. 98-101.

Noack AE, Coviella CE. Thaumastocoris australicus Kirkaldy (Hemiptera: Thaumastocoridae): first record this invasive pest of Eucalyptus in the Americas. General and Applied Entomology 2006; 35(2): 13-15.

Pereira JM, Melo APC, Fernandes PM, Soliman EP. Ocorrência de Thaumastocoris peregrinus Carpintero \& Dellapé (Hemiptera: Thaumastocoridae) no Estado de Goiás. Ciência Rural 2013; 43(2): 254-257. http://dx.doi. org/10.1590/S0103-84782013000200010.

Savaris MA, Lampert S, Pereira PRVS, Salvadori JR. Primeiro registro de Thaumastocoris peregrinus para o estado de Santa Catarina, e novas áreas de ocorrência para o Rio Grande do Sul, Brasil. Ciência Rural 2011; 41(11): 1874-1876. http://dx.doi.org/10.1590/S010384782011001100004

Soliman EP, Wilcken CF, Pereira JM, Dias TKR, Zaché B, Dal Pogetto MHFA et al. Biology of Thaumastocoris peregrinus in different eucalyptus species and hybrids. Phytoparasitica 2012; 40(3): 223-230. http://dx.doi. org/10.1007/s12600-012-0226-4.

Wilcken CF. Percevejo bronzeado do eucalipto Thaumastocoris peregrinus (Hemiptera: Thaumastocoridae): ameaça às florestas de eucalipto brasileiras. Botucatu: IPEF; 2008. Programa de Proteção Florestal - PROTEF/IPEF. Available from: http://www.ipef.br/protecao/alerta-percevejo.pdf.

Wilcken CF, Soliman EP, Nogueira de Sá LA, Barbosa LR, Dias TKR, Ferrera-Filho PJ et al. Bronze bug Thaumastocoris peregrinus Carpintero and Dellapé (Hemiptera: Thaumastocoridae) on Eucalyptus in Brazil and its distribution. Journal of Plant Protection Research 2010; 50(2): 201-205. http://dx.doi.org/10.2478/v10045010-0034-0

Wilcken CF, Soliman EP, Lima ACV, Dal Pogetto MHFA, Dias TKR. Perspectivas para o manejo do percevejo bronzeado do eucalipto. In: 14 Reunião técnica do PROTEF/IPEF; 2009; Botucatu. Botucatu: Faculdade de Ciências Agronômicas da UNESP; 2009. 